\title{
ON QUESTIONS OF B. J. GARDNER AND A. D. SANDS
}

\author{
K. I. BEIDAR \\ (Received 15 January 1991; revised 31 January 1992)
}

Communicated by P. Schultz

\begin{abstract}
An example of two disjoint special classes whose upper radicals coincide is presented. It is shown that the left hereditary subradical of the hereditarily idempotent radical is right hereditary. An example of a hereditary and principally left hereditary radical which is not left hereditary is constructed.
\end{abstract}

1991 Mathematics subject classification (Amer. Math. Soc.): 16 N 80.

All rings considered are associative. For the basic notions and results we refer to $[2,4,10,11]$. Let $\mathscr{P}$ be any class of rings and $\mathscr{Q}$ any class of prime rings. We shall make use of the following notations:

$\mathscr{S}(\alpha)$ - the semisimple class of the radical $\alpha$;

$\mathscr{U} \mathscr{P}$ - the upper radical generated by the class $\mathscr{P}$;

$\mathscr{L} \mathscr{P}$ - the lower radical generated by the class $\mathscr{P}$;

$\mathscr{E} S(\mathscr{P})$ - the essential cover of the class $\mathscr{P}$;

$\mathscr{S} d(\mathscr{P})$ - the subdirect closure of the class $\mathscr{P}$;

$\mathscr{A} c(\mathscr{P})$ - the class of all rings, which are isomorphic to accessible subrings of rings in $\mathscr{P}$;

$\mathscr{S}_{p}(\mathscr{Q})$ - the special class generated by the class $\mathscr{Q}$

A radical $\alpha$ is said to be hereditary (left hereditary, principally left hereditary) if $R \in \alpha$ and $I$ being an ideal (left ideal, $I=R r$ for some $r \in R$ ) of $R$ implies $I \in \alpha$.

In this paper we give affirmative answers to the following questions:

(C) 1994 Australian Mathematical Society 0263-6115/94 \$A2.00+0.00 
(1) Do there exist disjoint special classes $\mathscr{M}_{1}$ and $\mathscr{M}_{2}$ of prime rings with $\mathscr{U} \mathscr{M}_{1}=\mathscr{U} \mathscr{M}_{2}$ ? [6, p. 191].

(2) Does left-hereditary imply right-hereditary for subradicals of the class of hereditarily idempotent rings? [6, p. 193].

(3) Does there exist a hereditary principally left hereditary radical, which is not left hereditary? $[9$, p. 155].

\section{Examples of special classes}

LEMMA 1.1. If $\mathscr{P}$ and $\mathscr{Q}$ are classes of prime rings and $\mathscr{P} \subseteq \mathscr{S d}(\mathscr{Q})$, $\mathscr{Q} \subseteq \mathscr{S}_{d}(\mathscr{P})$, then $\mathscr{U}\left(\mathscr{S}_{p}(\mathscr{P})\right)=\mathscr{U}\left(\mathscr{S}_{p}(\mathscr{Q})\right)$.

PROOF. Clearly, $\mathscr{S}\left(\mathscr{U} \mathscr{S}_{p}(\mathscr{Q})\right)$ is a weakly special class. Hence $\mathscr{A}_{\mathcal{c}}(\mathscr{P}) \subseteq$ $\mathscr{S}\left(\mathscr{U} \mathscr{S}_{p}(\mathscr{Q})\right)$ and $\mathscr{E}_{s}\left(\mathscr{A}_{c}(\mathscr{P})\right) \subseteq \mathscr{S}\left(\mathscr{U} \mathscr{S}_{p}(\mathscr{Q})\right)$. But $\mathscr{S}_{p}(\mathscr{P})=\mathscr{E} S(\mathscr{A} c(\mathscr{P}))$, [8, Theorem 6]. Since $\mathscr{S}\left(\mathscr{U} \mathscr{S}_{p}(\mathscr{P})\right)=\mathscr{S} d\left(\mathscr{S}_{p}(\mathscr{P})\right)$ and $\mathscr{S}\left(\mathscr{U} \mathscr{S}_{p}(\mathscr{P})\right)=$ $\mathscr{S} d\left(\mathscr{S}_{p}(\mathscr{P})\right)=\mathscr{S}_{d}\left(\mathscr{E}_{s}(\mathscr{A} c(\mathscr{P}))\right) \subseteq \mathscr{S}_{d}\left(\mathscr{S}\left(\mathscr{U} \mathscr{S}_{p}(\mathscr{Q})\right)\right)=\mathscr{S}\left(\mathscr{U} \mathscr{S}_{p}(\mathscr{Q})\right)$, $\mathscr{S}\left(\mathscr{U} \mathscr{S}_{p}(\mathscr{P})\right) \subseteq \mathscr{S}\left(\mathscr{U} \mathscr{S}_{p}(\mathscr{Q})\right)$. Analogously, $\mathscr{S}\left(\mathscr{U} \mathscr{S}_{p}(\mathscr{Q})\right) \subseteq \mathscr{S}\left(\mathscr{U} \mathscr{S}_{p}(\mathscr{P})\right)$. Hence $\mathscr{S}\left(\mathscr{U} \mathscr{S}_{p}(\mathscr{P})\right)=\mathscr{S}\left(\mathscr{U} \mathscr{S}_{p}(\mathscr{Q})\right)$ and $\mathscr{U} \mathscr{S}_{p}(\mathscr{P})=\mathscr{U} \mathscr{S}_{p}(\mathscr{Q})$.

THEOREM 1.1. Let $C$ be an algebraic closure of the field $Q$ of rational numbers, $A=C\left[x_{1}, x_{2}, \ldots\right]$ be the $C$-algebra of polynomials over $C$ in commuting indeterminates $x_{1}, x_{2} \ldots, I$ be the $C$-ideal of $A$ generated by the polynomial $x_{1}^{2}+x_{2}^{2}-1$, and $B=A / 1$. Then:

(i) $\mathscr{U}\left(\mathscr{S}_{p}(A)\right)=\mathscr{U}\left(\mathscr{S}_{p}(B)\right)$;

(ii) $\mathscr{S}_{p}(A) \cap \mathscr{S}_{p}(B)=\{0\}$.

PROOF. For $n=2$ let $A_{n}$ be the subalgebra of $A$ generated by the elements $x_{1}, x_{2}, \ldots, x_{n}$. Put $I_{n}=I \cap A_{n}, B_{n}=A_{n} / I_{n}=\left(A_{n}+I\right) / I \subseteq B$ and $y_{i}=$ $x_{i}+I \in B$ for all $i=1,2, \ldots$.

We claim that $A \in \mathscr{S} d(B)$. Let $O \neq f \in A$. Clearly $f \in A_{n}$ for some $n \geq 2$. Let $\Phi: A \rightarrow B$ be a $C$-homomorphism such that $\phi\left(x_{n+1}\right)=y_{1}, \phi\left(x_{n+2}\right)=y_{2}$, $\phi\left(x_{i}\right)=y_{i+2}, \phi\left(x_{j}\right)=y_{j}$ for all $1 \leq i \leq n, j=n+3, n+4, \ldots$ Obviously, $\phi(f) \neq 0$. Hence $A \in \mathscr{S} d(B)$.

Further we claim that $B \in \mathscr{S} d(A)$. Obviously, $x_{1}^{2}+x_{2}^{2}-1$ is an irreducible polynomial in $C\left[x_{1}, x_{2}, \ldots\right]$. Hence for $n \geq 2, I_{n}$ is a prime ideal of $A_{n}$ and $B_{n}$ is an integral domain. Thus by Hilbert's Nullstellensatz $B_{n}$ is Jacobson 
semisimple and for every maximal $C$-ideal $M$ of $B_{n}, B_{n} / M$ is isomorphic to $C$. Let $0 \neq g \in B$. Clearly, $g \in B_{n}$ for some $n \geq 2$. Hence $g \notin M$ for some maximal $C$-ideal $M$ of $B_{n}$. Let $c_{1}, c_{2}, \ldots, c_{n}$ be the images of the elements $y_{1}, y_{2}, \ldots, y_{n}$ under the isomorphism $B_{n} / M \cong C$. Clearly, $c_{1}^{2}+c_{2}^{2}-1=0$. Define the $C$-endomorphism $\phi$ of $A$ by the rule $\phi\left(x_{i}\right)=c_{i}, \phi\left(x_{j}\right)=x_{j-n}$ for all $1 \leq i \leq n, j=n+1, n+2, \ldots$. Obviously, $\phi(I)=0$. Hence there exists a surjective homomorphism $\psi: B \rightarrow A$ such that $\psi(g) \neq 0$. Therefore $B \in \mathscr{S}_{d}(A)$. The foregoing and Lemma 1.1 show that $\mathscr{U} \mathscr{S}_{p}(A)=\mathscr{U} \mathscr{S}_{p}(B)$.

Suppose now that $0 \neq D \in \mathscr{S}_{p}(A) \cap \mathscr{S}_{p}(B)$. Since $\mathscr{S}_{p}(A)=\mathscr{E}_{s}\left(\mathscr{A}_{c}(A)\right)$ and $\mathscr{S}_{p}(B)=\mathscr{E}_{s}\left(\mathscr{A}_{\mathcal{C}}(B)\right)$, it follows that the ring $D$ has essential ideals $U$ and $V$ with $U \in \mathscr{A}_{c}(A)$ and $V \in \mathscr{A} c(B)$. Clearly $0 \neq U \cap V \in \mathscr{A}_{c}(A) \cap$ $\mathscr{A}_{C}(B)$. Hence the rings $A$ and $B$ have isomorphic accessible subrings $R$ and $S$, respectively. Let $\phi: S \rightarrow R$ be an isomorphism between the rings $S$ and $R$. Obviously, the isomorphism $\phi$ can be extended to an isomorphism $\phi: Q_{c l}(S) \rightarrow Q_{c l}(R)$ of quotient fields of $S$ and $R$. By [3, Lemma 4.2], $Q_{c l}(S)=Q_{c l}(B)$ and $Q_{c l}(R)=Q_{c l}(A)$. Thus $B \cong \phi(B) \subseteq Q_{c l}(A)$ and $R=\phi(S) \subseteq \phi(B)$. Hence $R$ is an accessible subring of $\phi(B)$. Now by [3, Lemma 4.2] there exists an integer $m \geq 1$ such that for all $r \in R, h \in \phi(B)$ and every integer $t \geq 1$, we have $r^{m} h^{t} \in R$. But $R \subseteq A$ and $A$ is a unique factorization domain. Hence $\phi(B) \subseteq A$, Further since $\phi(Q)=Q$, it follows that $\phi(C)=C$. Let $f=\phi\left(y_{1}\right)$ and $g=\phi\left(y_{2}\right)$. Then

$$
f^{2}+g^{2}-1=0 \text {. }
$$

Let $d: A \rightarrow A$ be the $C$-derivation of $A$ defined by $d\left(x_{1}\right)=1, d\left(x_{i}\right)=0$ for all $i \geq 2$. Further let $\operatorname{deg}(h)$ be the degree of the polynomial $h \in A$ with respect to the variable $x_{1}$. Since $\phi(C)=C$ and $y_{1}, y_{2} \notin C$, it follows that $f, g \notin C$. Without loss of generality we can assume that $\operatorname{deg}(f) \geq 1$. Now we have

$$
0=d\left(f^{2}+g^{2}-1\right)=2 f d(f)+2 g d(g)
$$

and

$$
f d(f)+g d(g)=0 .
$$

By (a) it follows that the polynomials $f$ and $g$ have no non-constant common divisor. Hence the polynomial $f$ divides the polynomial $d(g)$ and the polynomial $g$ divides the polynomial $d(f)$. Thus

$$
\operatorname{deg}(f) \leq \operatorname{deg}(d(g))<\operatorname{deg}(g) \leq \operatorname{deg}(d(f))<\operatorname{deg}(f)
$$

and we have a contradiction. Therefore $\mathscr{S}_{p}(A) \cap \mathscr{S}_{p}(B)=\{0\}$. 


\section{Left hereditary radicals}

THEOREM 2.1. Let $M$ be the class of all simple von Neumann regular rings with identity, $\beta$ be the lower Baer radical and $\alpha=\mathscr{L}[M \cup \beta]$. Then:

(i) $\alpha$ is hereditary and principally left hereditary;

(ii) $\alpha$ is not left hereditary.

PROOF. Let $I$ be an ideal of an $\alpha$-radical ring $A$. Suppose that $I \neq \alpha(I)$. It is well known that $\alpha(I)$ is an ideal of $A$ [1]. Let $M$ be an ideal of $A$ maximal with respect to the property $M \cap I=\alpha(I)$. Without loss of generality we can assume that $M=0$. Thus $\alpha(I)=0$. Since $\beta(A) \cap I \subseteq \alpha(I)=0$, it follows that $\beta(A)=0$. Further, since $\alpha=\mathscr{L}[M \cup \beta]$, it follows that the ring $A$ has an accessible subring $B$ which is a non-zero simple regular ring with an identity $e$. By [3, Lemma 4.3], $e$ is a central idempotent of the ring $A$ and $B=e A$. Hence $B$ is a non-zero ideal of $A$. Therefore $B \cap I \neq 0$. Since $B$ is a simple ring, it follows that $B \subseteq I$. Thus $B \subseteq \alpha(I)=0$ holds, a contradiction. Hence $I \in \alpha$ and so $\alpha$ is a hereditary radical.

Let $0 \neq A \in \alpha$ and $a \in A$. Suppose that $\alpha(A a) \neq A a$. Let $N$ be an ideal of the ring $A$ maximal with respect to the property $N \cap A a \subseteq \alpha(A a)$. Without loss of generality we can assume that $N=0$. Since $\beta(A) \cap A a \subseteq \alpha(A a)$, it follows that $\beta(A)=0$. Further, since $\alpha=\mathscr{L}[M \cup \beta], A$ has an accessible subring $D$ which is a non-zero simple regular ring with an identity $v$. By [3, Lemma 4.3] it follows that $v$ is a central idempotent of $A$ and $D=v A$. Clearly $D \cap A a \neq 0, D \cap A a=\{x \in A a \mid x v=x=v x\}=A v a=(v A) v a=D v a$ and $D \cap A a$ is an ideal of $A a$. Since $v a \in D$ and $D$ is a regular ring, it follows that $D v a=D u$ for some idempotent $u \in D$. Then $D u=u D u+(v-u) D u$. Clearly, $u D u$ is a simple regular ring with an identity $u$ and $(v-u) D u=\beta(D u)$. Hence $D u \in \alpha$ and $D \cap A a \in \alpha(A a)$ holds, contradicting the relation $N=0$. Therefore $A a \in \alpha$ and so $\alpha$ is principally left hereditary.

Let $A$ be a simple regular non-artinian ring with identity and $M$ be a maximal left ideal of $A$. By [3, Theorem 1] $M$ is a simple ring. Suppose that $M \in \alpha$. Then $M$ must be a simple regular ring with identity. Let $e$ be an identity of $M$. Then $A e=(A e) e \subseteq M e \subseteq A e$ and $A e=M e=M$. Since $M$ is a maximal left ideal of $A$, it follows that $A(1-e)$ is a minimal left ideal of $A$. Hence the socle, $\operatorname{Soc}(A)$, of $A$ is non-zero. But $\operatorname{Soc}(A)$ is an ideal of $A$, so, since $A$ is simple, $A=\operatorname{Soc}(A)$. Further since the ring $A$ has an identity, it follows that $A$ is a finite direct sum of minimal left ideals. Hence the ring $A$ is artinian, a contradiction. Therefore $M \notin \alpha$. Clearly $A \in \alpha$. Hence the radical $\alpha$ is not left 
hereditary.

Recall that a ring $A$ is said to be strongly regular if for every $a \in R$ there exists $x \in R$ with $a=x a^{2}$ (see [5]). By [8, Theorem 3.5], a strongly regular ring is a regular ring, all of whose idempotents are central. Let us denote by $A^{\sharp}$ the ring $A$ with an identity adjoined.

THEOREM 2.2. For a radical $\alpha$ the following conditions are equivalent:

(i) $\alpha$ is a left hereditary subradical of the class of all hereditarily idempotent rings:

(ii) $\alpha$ is a right hereditary subradical of the class of all hereditarily idempotent rings;

(iii) every $\alpha$-radical ring $A$ is a strongly regular ring.

ProOF. (i) implies (iii). Let $A \in \alpha$. Observe that for every $a \in A, L=A^{\sharp} a$ is a left ideal of $A$ and $M=Z a+A^{\sharp} a^{2}$ is a left ideal of $L$, where $Z$ is the ring of integers. Thus $M \in \alpha$ and consequently $M^{2}=M$. This means that $a=x a^{2}$ for some $x \in A^{\sharp}$. Hence $a=x a^{2}=x\left(x a^{2}\right) a=\left(x^{2} a\right) a^{2}$. Since $y=x^{2} a \in A$ and $a=y a^{2}, A$ is a strongly regular ring.

(iii) implies (i). Let $A \in \alpha$ and $L$ be a left ideal of $A$. Further let $a \in L$. Since $A$ is a strongly regular ring, it follows that $A a=A e$ for some central idempotent $e \in A$. Hence $A e$ is an ideal of $A$ and $A=A e \oplus A(1-e)$. Therefore $A e \in \alpha$ and $a \in A e \subseteq \alpha(L)$ which means that $\alpha$ is a left hereditary radical.

Symmetric arguments show that (ii) implies (iii) and (iii) implies (ii).

\section{Acknowledgements}

The author gratefully acknowledges the financial support of the Hungarian National Foundation for Scientific Research Grant No. 1813. He also expresses his thanks to Professor Wiegandt for the kind help he gave the author in writing this paper, and to Professor Puczylowski and the referee for valuable suggestions, which simplified the proof of Theorem 2.1.

\section{References}

[1] T. Anderson, N. Divinsky and A. Sulinski, 'Hereditary radicals in associative and alternative rings', Canad. J. Math. 17 (1965), 594-603. 
[2] V. A. Andrunakievich and Ju. M. Ryabukhin, Radicals in algebras and structural theory (Nauka, Moscow, 1979) (in Russian).

[3] K. I. Beidar, 'Atoms in the lattice of radicals', Mat. Issled. 85 (1985), 21-31 (in Russian).

[4] N. Divinsky, Rings and radicals (Allen and Unwin, London, 1965).

[5] A. Forsythe and N. H. McCoy, 'On the commutativity of certain rings', Bull. Amer. Math. Soc. 52 (1946), 523-526.

[6] B. J. Gardner (ed.), Rings, modules and radicals, (Proceedings of the Hobart Conference, 1987), Research Notes in Mathematics 204 (Pitman, London, 1989).

[7] K. R. Goodearl, Von Neumann regular rings (Pitman, London, 1979).

[8] G. A. P. Heyman and C. Roos, 'Essential extension in radical theory of rings', J. Austral. Math. Soc. (Series A) 23 (1977), 340-347.

[9] A. D. Sands, 'Radical properties and one sided ideals', in: Contribution to general algebra 4, Proceedings of the Krems Conference 1985 (Hölder-Pichler-Temsky, Vienna, 1987) pp. 151-171.

[10] F. A. Szász, Radicals of rings (Akadémiai Kiad́o, Budapest, 1981).

[11] R. Wiegandt, Radical and semisimple classes of rings, Queen's papers in pure and applied mathematics 37 (Kingston, Ontario, 1974).

Department of Algebra

Moscow State University

117234 Moscow, USSR 